\title{
Why \\ Are \\ There No 3-Headed Monsters? Mathematical Modeling in Biology
}

J. D. Murray

A lan Turing's crucial intelligence work in the Second World War is well known. His contribution to the interdisciplinary field of mathematics and the biological sciences is less so. Turing published only one paper related to biology, "The chemical basis of morphogenesis", in 1952 [64], which has been seminal in several areas of spatial patterning modeling in development, ecology, and other biological areas since its rediscovery in the 1960s. He did not apply his model to any specific biological situation.

Basically Turing showed how, in a system of reacting chemicals where the chemicals can also diffuse, the system can generate a steady-state heterogeneous spatial pattern of chemical concentrations. He called these chemicals morphogens. He hypothesized that these morphogenetic prepatterns could cue cell differentiation and result in observed spatial patterns. His model is encapsulated in the coupled system of reaction diffusion equations, of the general form

(1)

$\frac{\partial u}{\partial t}=\gamma f(u, v)+D_{u} \nabla^{2} u, \frac{\partial v}{\partial t}=\gamma g(u, v)+D_{v} \nabla^{2} v$,

where the functions $f(u, v)$ and $g(u, v)$ denote the reaction kinetics associated with the chemicals $u$, called the activator, and $v$, called the inhibitor, with $D_{u}$ and $D_{v}$ the diffusion coefficients of $u$ and $v$ respectively. The parameter $\gamma$, which arises

J. D. Murray is professor emeritus of mathematical biology, University of Oxford; senior scholar in applied and computational mathematics, Princeton University; and emeritus professor of applied mathematics, University of Washington. His email address is james . mu r ray@maths . ox. ac.uk and jdmurray@princeton. edu.

DOI: http://dx.doi.org/10.1090/noti865 when the system is written in this nondimensional form, is an important measure of scale, as we shall see below. A stability analysis of the steady states of the kinetics shows that to generate spatial patterns in $u$ and $v$, it is necessary, among other things, that the inhibitor have a higher diffusion rate than the activator, that is $D_{v}>D_{u}$; see, for example, [44], [46]. A review article [35] is specifically devoted to Turing's theory.

To get an intuitive idea of how the reaction diffusion patterning works, consider the following, albeit unrealistic scenario, of a field of dry grass in which there is a large number of grasshoppers. If grasshoppers get warm, they can generate a lot of moisture by sweating. Now suppose the grass is set alight at several random points and a flame front starts to propagate from each. We can think of the grasshopper as an inhibitor and the fire as an activator. If there were no moisture to quench the flames, the fires would simply spread over the whole field, which would result in a uniform charred area. Suppose, however, that when the grasshoppers get warm enough they can generate enough moisture to dampen the grass so that when the flames reach such premoistened areas the grass will not burn. The scenario for a heterogeneous spatial pattern of charred and uncharred grass patches is then the following. The fires start to spread; these represent one of the "reactants", the activator, with a fire "diffusion" coefficient, which quantifies how fast the fire spreads. The grasshoppers, which constitute the inhibitor "reactant", feel the flame fronts coming and move quickly ahead of them. The grasshoppers then sweat profusely and generate enough moisture to prevent the fires spreading into the moistened area. In this way the charred areas 
are restricted to finite domains that depend on the "diffusion" coefficients of the reactants-fire and grasshoppers - and the various "reaction" parameters. If the grasshoppers and flame fronts "diffused" at the same speed, no such spatial pattern could evolve.

Closely related to Turing's theoretical work, and done independently, is the important experimental work of Boris Belousov. Although the exact date is not known, it was in the early 1950s that he showed how a group of three reacting chemicals could spontaneously oscillate between a colorless and a yellow solution. His paper was twice rejected for publication, and he was eventually persuaded to publish it in an obscure journal of abstracts in 1959. This seminal work was rediscovered by Anatol Zhabotinsky in 1961 [69], and this classic groundbreaking reaction, known as the Belousov-Zhabotinsky reaction, has been widely studied experimentally (see, for example, [52]) and mathematically (see, for example, [44]).

A vast number of papers refer to such oscillating systems and to reactions that exhibit oscillatory behavior and generate complex spatial patterns: see, for example, the early work in 1972 [66] that shows how spiral waves of reactants arose. The books [44], [46] discuss the reaction diffusion systems in detail. There are several webpages that visually demonstrate these oscillating reactions and their spatial patterning.

There are several classic examples of the mathematical models. One very early one appears in the little-known paper (in French) [6], written by Bernoulli in 1760, when smallpox was rampant. He proposed a differential equation model to quantify how cowpox inoculation affects the spread of smallpox. As well as providing an interesting and practical mathematical model, the article gives some interesting data on the devastating child mortality at the time. He used the model to assess the practical advantages of a vaccination control program. One wonders if he knew of the ancient Chinese custom where children were made to inhale a powder made from the crusts of skin lesions of people recovering from smallpox. A few researchers in the first half of the twentieth century were getting involved in mathematical modeling associated with population interaction and epidemic models, but they seem not to have known about Bernoulli's remarkable paper [15], [26], [27], [32], [65]. A major interdisciplinary work, albeit not specifically mathematical but heuristically so, is D'Arcy Thompson's [63] monumental book On Growth and Form, first published in 1917, which discusses among other things concepts associated with morphological patterns. The rediscovery of Turing's 1952 paper in the late 1960s had a major influence in the development of the field of mathematical biology, surprisingly more than the experimentally justified model of Hodgkin and Huxley published in 1952 [22] on nerve conduction and excitation, which was awarded a Nobel Prize.

Considerable interest in the study of pattern formation had been developing by the time the remarkable work of Geoffroy Saint-Hilaire appeared in 1836 [55]. He was particularly interested in teratology and was probably the first to introduce the important concept of a developmental constraint, something we shall talk about below. His emphasis on the parallels between the study of form in physical systems and of biological form was an early indication of later papers in the field.

Until around 1970 mathematical involvement in the biomedical sciences was restricted to just a few researchers who developed predator-prey models, basic epidemic models and others associated with problems in body mechanics, facilitated diffusion in tissues [37], and others. The use of mathematics in biology really started to blossom from the mid-1970s, with several thousand researchers now actively involved and mathematical modeling being used in practically every field in the biomedical sciences.

Much of the research in the twentieth century in the applications of mathematical modeling in biology was in biological pattern formation, a field about which there was no general acceptance but a lot of controversy. With the burgeoning of genetic studies, the belief that genetics would solve all these developmental problems has certainly not been borne out. There was, of course, considerable research in the ecological and epidemiological sciences, where the use of mathematical models had a long history.

In the past twenty to thirty years, genetic studies have spawned exciting, important, and genuinely interdisciplinary research involving theoreticians and experimentalists, with the common aim of elucidating the underlying mechanisms involved in developmental biology and medicine. However, most of the mechanisms are essentially still unknown.

This interdisciplinary field is nowadays referred to as mathematical biology, theoretical biology, or systems biology. An increasing number of books provide surveys of some of the early work in the field as well as perspectives on the field's remarkable growth since the 1970s; examples include [37], [44], [46] and the definitive text [25] on the relatively recent growth area of modeling in physiology. There are many other books with author-contributed chapters, often associated with conference proceedings, that give a picture of how the field developed, such as [7], [11], [23], [29], [31], [33]. The book [36] is specifically 
devoted to reaction-diffusion theory. Research on theoretical models in ecology and epidemiology has also been growing; see, for example, the 1992 review [30]. With the ever-increasing number of people getting involved in the field, the number of genuinely practical examples has grown, as has the number of essentially mathematics papers where the primary interest is in the mathematics, with scant connection to any real biology. By the mid-1980s it was becoming more widely acknowledged that any real contribution to the biological sciences from modeling must be genuinely interdisciplinary and hence related to real biology. The best research helps our understanding of real biological problems by developing models for specific biological situations and providing predictions that are confirmed (or contradicted) by experiment; a specific example is discussed below.

A significant influence on the development of mathematical biology came from the seminal experimental work on the importance of chemical gradients in embryonic development [67], which introduced the concept of "positional information". The experiments showed that cells react to a chemical concentration associated with where they are in a chemical gradient. This work stimulated a huge amount of experimental and theoretical work, often controversial, that is still going on. For a review, see the book [68] on the author's views on the principles of development.

Since the late 1990s there has been an everincreasing number of truly interdisciplinary studies covering a remarkably wide spectrum of topics. One example is research on wound healing [14], [16], [56], [57]. Another is on tumor growth, such as brain tumors (see [47] for a short review), briefly described below, which is being used medically to quantify the efficacy of individual patient treatment scenarios prior to treatment (see [46] for a survey).

Many of these cancer studies involve modeling the phenomena across many different scales. One example is the seminal work [17]. The suggestion here was that tumor cells create an environment that allows certain mutations to be selected and hence the evolution of mutant cell populations to occur within the body; this is called somatic evolution. The authors analyzed somatic evolution in this context and showed a number of evolutionary pathways in ductal carcinoma in situ. The experimental colleagues suggested that different mutant clones would emerge in a well-defined temporal sequence, while the mathematical simulations showed that this was highly unlikely. The simulations predicted that hypoxia (lack of oxygen) should promote emergence of varyingsized nodules of a mutant clone of a certain type surrounded by other cell types and that over time these nodules would grow and merge. This stimulated the experimental colleagues to carry out experiments, which confirmed this prediction. Another study highlights cellular adaptions of cancer cells in colorectal cancer [24]. In the following section we briefly discuss one application of reaction-diffusion theory. We shall point out some of the limitations of Turing-type reactiondiffusion mechanisms that necessitated a new, and more experimentally verifiable, approach to biological pattern formation, known as the mechanical theory of biological pattern formation [40], [41], [50].

\section{How the Leopard Gets Its Spots}

A specific experimental reaction-diffusion mechanism proposed in 1975 [62] was used to study how animal coat patterns might be formed [38], [39], [43], [44]. The reaction terms used in (1) were

$$
\begin{aligned}
& f(u, v)=a-u-h(u, v), \\
& g(u, v)=\alpha(b-v)-h(u, v), \\
& h(u, v)=\frac{\rho u v}{1+u+K u^{2}},
\end{aligned}
$$

where $a, b, \alpha, \rho$, and $K$ are constants and assigned so that the solutions exhibited steady-state spatially heterogeneous solutions. They were kept fixed for all the calculations. Only the scale and geometry of the domain were varied. The resulting patterns in Figures 1 and 2 are colored dark and light in regions where the concentration of one of the morphogens is greater than or less than the concentration in the homogeneous steady state. Even with such limitations on the parameters, the wealth of possible patterns is remarkable. For a given domain size and geometry each set of initial conditions gave a similar but unique pattern, a fact reflected in nature.

It was shown that a single prepatterning mechanism was capable of generating the typical geometry of mammalian coat patterns, from the mouse to the badger to the giraffe to the elephant and almost everything in between, with the end pattern governed simply by the size and shape of the embryo at the time the pattern formation process was initiated. In solving these reaction-diffusion systems, the domain size and shape are crucial. If, given a particular mechanism, one tries to simulate a solution in a very small domain, one finds that it is not possible to obtain steady-state spatial patterns; a minimum size is needed to drive any sustainable spatial pattern. Or, if the domain is long and thin, only stripes can be generated.

Suppose the reaction-diffusion domain is a rectangle. The rectangle must have a minimum size for a simulation to exhibit spatial heterogeneity. As the size of the rectangle is increased, a series of 




(a)

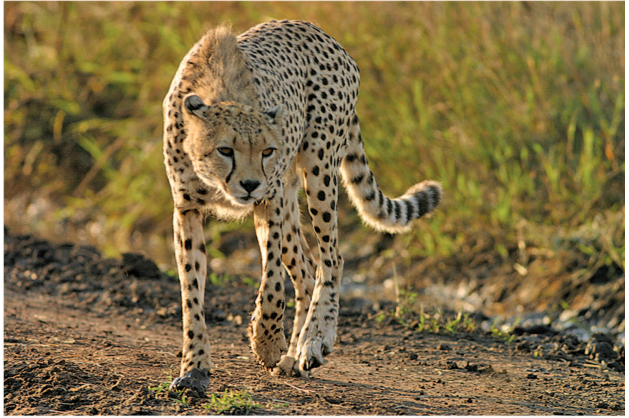

(b)

Figure 1. (a) Examples of a developmental constraint. Spotted animals can have striped tails but not the other way round. From left to right are the typical tail of the leopard, of the cheetah, and of the genet, together with the solutions from a reaction-diffusion system which can generate steady-state spatial patterns. The geometry and scale when the pattern mechanism is activated play crucial roles in the resulting coat patterns. Dark regions represent areas of high morphogen concentration. (Tail art work reproduced from [43] with permission of Patricia Wynne.) (b) A cheetah (Acinonyx jubatus), which is an example of the developmental constraint described in (a). (Photograph courtesy of Professor Andrew Dobson.)

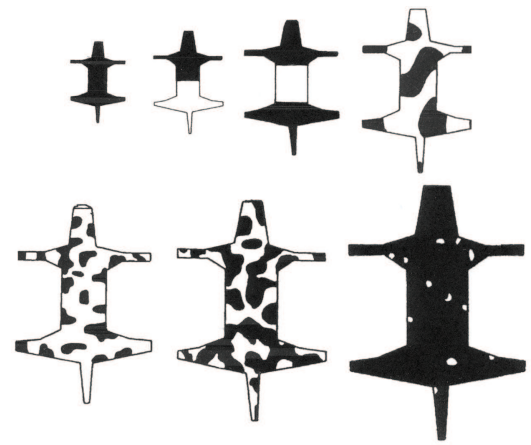

(a)



(b)



(c)

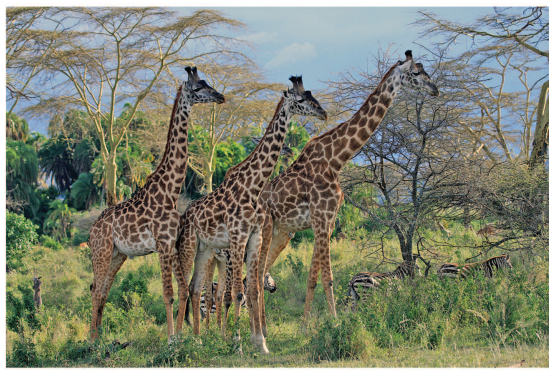

(d)

Figure 2. (a) These show the result of numerical simulations of the reaction-diffusion model analysis [38], [39], [43], [44] for the generation of coat markings on animals; it is the same mechanism used in Figure 1. The model parameters were also the same; only the scale parameter was varied. The domain sizes have been reduced to fit in a single figure, but in the simulations there was a scale difference of

1,000 between the smallest and the largest figure. (b) An example of the first bifurcation: Valais Blackneck goat. (Photograph by B. S. Thurner Hof, Wikimedia Commons.) (c) Belted Galloway cows. (d) Giraffes (Giraffa camelopardis) in the Serengeti. (Photograph courtesy of Professor Andrew Dobson.) 
increasingly complex spatial patterns emerge. The concept behind the model is that the simulated spatial patterns, solutions of a reaction-diffusion mechanism, reflect the final morphogen melanin landscape observed on animal coats. With this scenario, the cells react to a given level in morphogen concentration, thus producing melanin (or rather becoming melanocytes, cells that produce melanin). In Figures 1(a) and 2(a), the black regions represent high levels of melanin concentration. It should be emphasized that this model is a hypothetical one that has not been verified experimentally but rather circumstantially. The main purpose is to show how scale and shape play major roles in animal coat patterns, as they must in other developmental processes. Such an approach has also been used to study butterfly wing patterns [38], [39], [44], [49]. Importantly, the work [49] presents experimental confirmation of the theoretical predictions.

The solutions of the reaction-diffusion system (1) and (2) in domains shown in Figure 1(a) were first computed as an example of how the geometry constrains the possible pattern modes. When the domain is very narrow, only simple, essentially one-dimensional modes can exist. Twodimensional patterns require the domain to have enough two dimensionality. Consider a tapering cylinder as in Figure 1(a). If the radius at one end is large enough, two-dimensional patterns can exist on the surface. So, a tapering cylinder can exhibit a gradation from a two-dimensional pattern to simple stripes, as illustrated in Figure1(a).

This shows that the conical domain mandates that it is not possible to have a tail with spots at its tip and stripes at its base, but only the converse; Figure 1(a) shows some examples of specific animal tails. This is a genuine example of a developmental constraint. The cheetah, a photo of which is shown in Figure 1(b), is a prime example of this, as are other spotted animals. If the threshold level of the morphogen is changed, a different but related pattern can develop. Such mechanisms can thus form different but globally similar patterns and could be the explanation for the different types of spatial patterns observed on different species of the same animal genre, such as the spots of the cheetah in Figure 1(b) and the spots of the giraffe in Figure 2(d).

The interpretation of Figure 2 is that if the animal embryo is too small when the patterning mechanism is activated, as in the mouse, or too large, as in the hippopotamus and elephant, then no clear pattern will be observed and these animals are essentially uniform in color.

In between there is a progressively more complex pattern as the size increases. The first two bifurcations are illustrated in Figures 2(a) and 2(b), with the larger animals still showing coat pattern but looking progressively uniform in color, as shown in Figure 2(a).

There have been numerous developments and an increased understanding of how patterns on animals-fish and butterflies, for example-are formed with the addition and combination of other pattern-forming mechanisms, such as chemotaxis, whereby there is movement of cells up chemical gradients. Some examples of the numerous review articles and books are [5], which discusses somite formation; [53], which discusses fish stripes; [28], which discusses evolving fish patterns and other patterned species [10], [34], [35], [59].

It is pointed out in [4] that an understanding of the diversity of animal coat patterns requires an understanding of both the mechanisms that create them and importantly their adaptive value. Among other things, this paper discusses the advantages of specific patterns in different environments. The authors use a reaction-diffusion model, but their conclusions are general and do not rely on any specific reaction-diffusion models, as is essentially the case with the above. They convincingly show how different markings relate to specific natural environments for the specific fields.

The areas of application of reaction-diffusion models are now legion. One particular simple application, which has turned out to be surprisingly practical, was initially proposed in 1995 [9] and pertains to the growth of gliomablastoma brain tumors. The model is given by

$$
\frac{\partial c}{\partial t}=\nabla \cdot D(x) \nabla c+\rho c .
$$

Here $c(\mathbf{x}, t)$ is the cancer cell density, measured in cells $/ \mathrm{mm}^{3}$, at position $\mathbf{x}$ in the brain at time $t$ measured in months; $D(\mathbf{x})$ is the cell diffusion (invasion), measured in $\mathrm{mm}^{2} /$ month, which quantifies the invasiveness of the cancer cells at position $\mathbf{x}$ in the brain, since it varies depending on position; and $\rho$ is the net proliferation rate (/month) of the cancer cells, which gives the cell turnover time as $\log 2 / \rho$ (months). It is possible with this model and patient brain scans to quantify the efficacy of various treatment scenarios for individual patients prior to treatment [60], [61]. These tumors are always fatal: see [46] for a full discussion and [47] for a recent brief review.

\section{Mechanical Theory of Biological Pattern Formation in Morphogenesis}

Because of the paucity of experimental verification, the limitations of reaction-diffusion theory gave rise to a totally new theory of biological pattern formation (exceptions are ecology and epidemiology; see, for example, [18], [29] for reviews). The theory was based on extant biological facts about cells and 


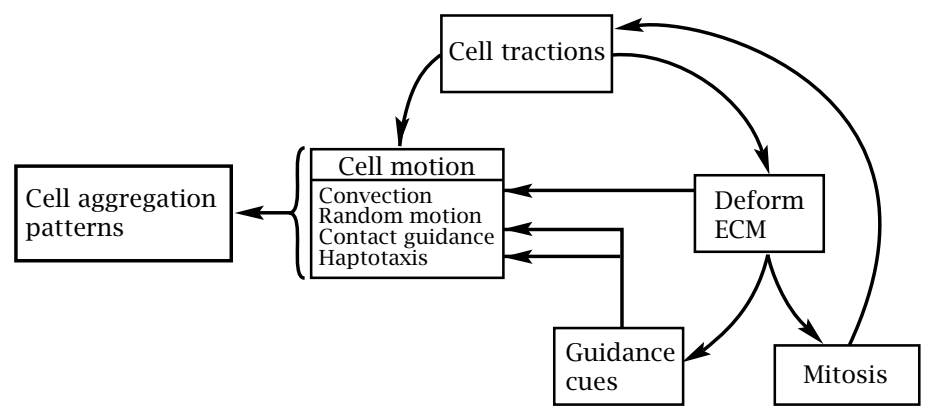

Figure 3. Mechanical theory patterning scenario. Here ECM denotes the extracellular matrix through which the cells move, mitosis is cell division, and haptotaxis is cell movement directed by the deformed biological matrix.

the extracellular tissue matrix, namely, the OsterMurray mechanical theory of pattern formation [40], [41], [50], [51]. A short introduction is given in [42] with a full survey in [44], [46]. The class of models captures the key interactions between the mechanical forces generated by the cells and their extracellular matrix milieu. Together they give rise to developmental processes that could be experimentally verified. The model shows that a purely mechanical version of the theory could be responsible for certain observed patterns and how they are actually formed in development. Such mechanical models are based on basic mechanical concepts and do not specify the type of cells and matrix involved but instead consider only possible mechanical interactions between the various components.

The basic model hinges on two key experimentally determined properties of mesenchymal cells in vivo: (i) cells migrate within a tissue substratum made up of fibrous extracellular matrix, the ECM; (ii) cells can generate large traction forces, thereby deforming the matrix. The basic mechanism models the mechanical interaction between the motile cells and the elastic substratum within which they move. Mesenchymal cells move by exerting forces on their surroundings, consisting of the elastic fibrous ECM and the surface of other cells. They use their cellular protrusions, which stretch out from the cell in all directions, gripping whatever is available and pulling. Due to the heterogeneity in matrix and cell densities, cell traction tension lines form between the cell clusters. These tension lines correspond to aligned matrix fibers along which cells actively move, thereby defining cellular highways between the clusters. One of the major roles of the modeling and its analysis was to indicate what features are essential for biological pattern formation.

The models consist of three nonlinear partial differential equations; the cell conservation equation; another for the cell-extracellular tissue interaction, which incorporates cell movement, that is, mechanotaxis or haptotaxis; and the third equation, which quantifies the cell-matrix mechanical interaction. The models pose numerous challenging mathematical problems, both analytical and numerical, as well as biological modeling problems, many of which have not yet been investigated in any depth. Although the model system is analytically formidable, its conceptual framework is quite clear, as illustrated in Figure 3. The parameters in the model equations are all quantifiable from experiment.

Several factors affect the movement of embryonic mesenchymal cells. Among these are: (i) convection, whereby cells may be passively carried along on a deforming substratum; (ii) diffusion, where the cells move randomly but generally down a cell density gradient; (iii) contact guidance, in which the substratum on which the cells crawl suggests a preferred direction; (iv) contact inhibition by the cells, whereby a high density of neighboring cells inhibits motion; (v) haptotaxis, where the cells move up an adhesive gradient; (vi) chemotaxis, whereby a chemical gradient can direct cell motion both up and down a concentration gradient; (vii) galvanotaxis, where movement from the field generated by electric potentials, which are known to exist in embryos, provides a preferred direction of motion. These effects are all well documented from experiment. The analysis of the field equations incorporating only (i)-(v) showed how regular spatial aggregates of cells come about.

\section{Evolution and Morphogenetic Rules in Cartilage Formation in the Vertebrate Limb: Why Are There No 3-Headed Monsters?}

One major application of this mechanical theory was to limb development, the results of which were also put into an evolutionary context [51]. Since the limb is one of the most morphologically diversified of the vertebrate organs and one of the more easily studied developmental systems, it is not surprising it is so important in both embryology and evolutionary biology, where there is a rich fossil record documenting the evolution of limb diversification.

Although morphogenesis appears deterministic on a macroscopic scale, on a microscopic scale cellular activities during the formation of the limb involve considerable randomness. Order emerges as an average outcome with some high probability. It was shown that some morphogenetic events are extremely unlikely, such as trifurcations from a single chondrogenic condensation. Mathematically, of course, they are not strictly forbidden by the pattern formation process but are highly unlikely, since they correspond to a delicate choice of 


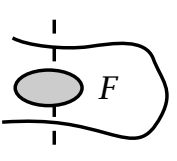

(a)



(b)



(c)

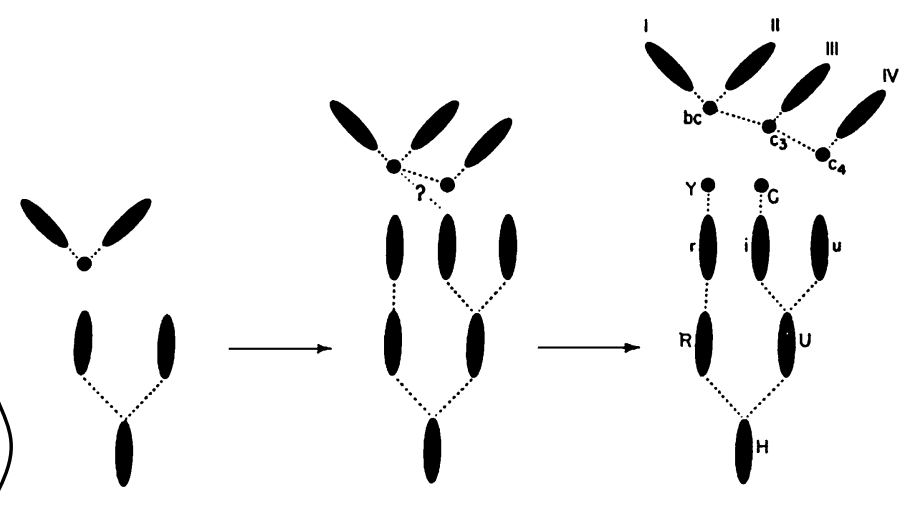

(d)

Figure 4. Morphogenetic rules. The three basic cell condensations: (a) a single condensation, $F$; (b) a branching bifurcation, $B$; (c) a segmental condensation, $S$. Complicated patterns can be built from a combination of these basic bifurcations, as in (d), which is one example of the complex morphogenesis and digitations of the forelimb of the salamander Ambystoma mecicanum, which was obtained experimentally [44], [58].

conditions and parameter tuning. This is another experimentally verified example of a developmental constraint. The "morphogenetic rules" for limb cartilage patterning are summarized in Figure 4.

A variety of limb buds were treated with the mitotic inhibitor colchicines [3]. This chemical reduces the dimensions of the limb by reducing cell proliferation. It was predicted from the mathematical model that such a reduction in tissue size reduces the number of bifurcation events. Figure 5 quantitatively shows the consequence of the inhibitor on limb cartilage growth.

Using the basic rules of cartilage pattern formation in [3], a series of comparative studies [58] were carried out with amphibians, reptiles, birds, and mammals, which confirmed the mathematical predictions, or hypothesis, that tetrapod limb development consists of iterations of the processes of focal condensation, segmentation, and branching. Furthermore, it was shown that the patterns of precartilage cell condensation display several striking regularities in the formation of the limb pattern. The experimental results supported the theoretical conclusion that branching, segmentation, and de novo condensation events are reflections of the basic cellular properties of cartilage-forming tissue.

We thus see the possibility of evolution moving backward. It is clearly possible when we consider evolution of form as simply variations in mechanical (or rather mechanistic) parameters. Figure 5 is an unequivocal example where this has happened solely through changing the morphogenetic processes.

The study of these theoretical mechanical models for pattern formation showed that there are considerable restrictions as to the possible patterns of chondrogenesis (as well as other developmental aspects). From the morphogenetic laws it is highly unlikely that a trifurcation is possible, that is, a branching of one element into three elements. There are numerous examples of two-headed snakes and other reptiles, conjoined twins, and so on. Although there is sometimes an appearance of a 1-to-3 splitting, the theory suggests that all branchings are initially binary. This is because a trifurcation is possible only under a very narrow set of parameter values and conditions. Including asymmetries makes it even more unlikely. This notion of the unlikelihood of trifurcations is the reason we do not see any three-

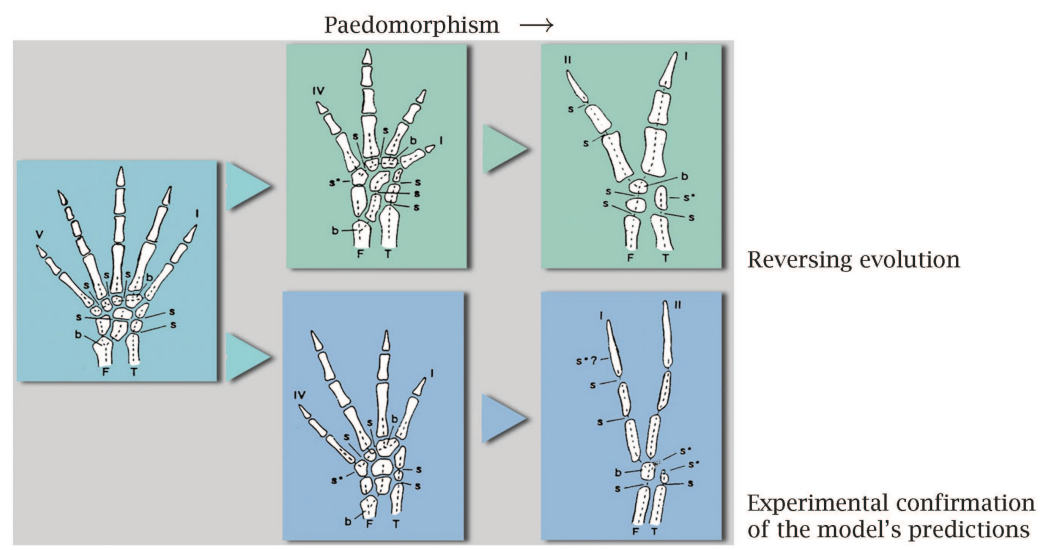

Figure 5. The effect of treating the foot of the salamander Ambystoma mexicanum with the mitotic inhibitor colchicine is to reduce the number of skeletal elements. The effect of the inhibitor is to reduce the cell number in the limb and hence the size (after [1], [2]). 




(a)

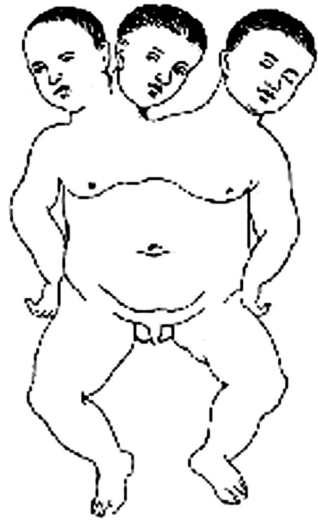

(b)
Figure 6. Examples of branching in humans. (a) The skeleton (19th century) of a Dicephalus, a young boy. (b) An example (19th century) of a Tricephalus.

headed monsters. Figure 6 shows two examples of human branching.

Very few three-headed monsters have been reported, and of these the veracity is usually highly questionable. If we come back to the limited bifurcations suggested by the morphogenetic laws above, and specifically that a trifurcation is highly unlikely, we can see how a three-headed monster can arise, namely, via a bifurcation of the body axis, such as we see in the skeleton in Figure 6(a), followed by a further bifurcation of one of the branches, as seems clear in the example in Figure 6(b). See [46] for a very brief history of writings on monsters.

Teratology highlights some of the most fundamental questions in evolution: namely, why do we not get certain forms in nature? The developmental process embodies various systems of constraint that bias the evolution of the system. Teratologies, among other things, provide an excellent source of information on the potential of developmental processes. They also suggest which monstrosities are possible and which are not. It is interesting that specific morphologies are found in quite different species, suggesting a certain common developmental process for part of their development.

\section{Concluding Discussion}

This has been a very short and personal choice from the vast literature associated with the application of mathematical models in the biomedical sciences. In the 1980s, with most of the research conclusions speculative, there was a decrease in the new applications of reaction-diffusion models, since demonstrating the existence of specific morphogens was proving elusive. This resulted in the new mechanochemical theory of biological pattern and form discussed briefly above, which was based on experimental data on real cells and the forces they could exert in the generation of pattern and form. From the mid-1990s on, the practical use of reaction-diffusion models again increased, with the biological applications becoming much more verifiable experimentally, as has research and developments of the Murray-Oster mechanochemical theory of pattern formation as more experimental data and confirmation of modeling predictions have been found.

As seen in many of the papers referred to here, models and their biological predictions encouragingly have been a major stimulant for guiding critical experiments, which have resulted in significant discoveries. This, of course, should be the aim of any mathematical biology modeling, namely, to stimulate in any way whatsoever any endeavor that results in furthering our understanding of biology. Although with the major developments in the past twenty years we now know a lot more about pattern development, most mechanisms involved in development are still not fully understood. We do not know, for example, the complete mechanisms of how cartilage patterns in developing limbs are formed or the specialized structures in the skin, such as feathers, scales, glands, and hairs, or the myriad of widely observed patterns. Many of the rich spectrum of spatial patterns observed in development evolve from a homogeneous mass of cells that are orchestrated by genes that initiate and control the pattern formation mechanisms; genes themselves are not involved in the actual physical process of pattern generation. The basic philosophy behind practical modeling in biology is to try to incorporate the physicochemical events, which from observation and experiment appear to be going on during development, within a model mechanistic framework that can then be studied mathematically and, importantly, the results related back to the biology. These morphogenetic models provide the embryologist with possible scenarios as to how and when pattern is laid down, how elements in the embryo might be created, and what constraints on possible patterns are imposed by different models. Many of the references in this article have greatly increased our biological understanding.

Both the mechanochemical models and reaction-diffusion models have been fruitfully applied to a vast range of biological problems, not only in morphogenesis but elsewhere, such as feather primordia arrangement, wound healing mentioned above, wound scarring, shell and mollusk patterns, and many others [46]. It is almost certain that both mechanisms are involved in development, and although they 
are in a sense competing theories, in fact the mechanisms complement each other. Perhaps the most fundamental difference between the theories is that the elements involved directly in the mechanical theory are all real biological quantities: namely, cells, tissue, and the forces generated by the cells. All quantities involved are measurable. In the end, however, the key aspect of these mechanisms is their ability to predict the subsequent pattern and form. The final arbiter of a model's correctness and usefulness is how consistent it appears in the light of subsequent experiments and observations.

The explosion in biochemical techniques over the last several decades has led to a still larger increase in our biological knowledge but has partially eclipsed the study of the intermediate mechanisms that translate gene influence into chemicals, into gradients, and into pattern and form. As a result, there is much still to be done in this area, both experimentally and theoretically.

We have clearly only scratched the surface of a huge, important, and ever-expanding interdisciplinary world. Biology, in its broadest sense, is clearly the science of the foreseeable future. What is clear is that the application of mathematical modeling in the biological, medical, ecological, psychological, and social sciences is going to play an increasingly important role in future major discoveries and epidemiological and population control strategies. There is an ever-increasing number of areas where theoretical modeling is important, such as social behavior, adaption to habitat changes, climate change, and so on. In the case of zebras, for example, in [54] it is shown, by unraveling how species adapt to specific environmental changes such as land use, why of two types of zebra in the same environment the Grevy zebra (Equus grevyi) is nearing extinction, while another, the plains zebra (Equus burchelli), has adapted its behavior to survive. Behavioral ecology is another important expanding area of research. How bird flocks, schools of fish, and so on reach community decisions is another exciting, relatively new area; see [48] for research on fish community decisions and [8] for locusts.

Mathematical biology now has active researchers, numbering in the thousands, in practically all of the biomedical sciences. Mathematical modeling in the social sciences is another growth area of the future. One example of this involvement is the theoretical model developed for a major study on marital interaction and divorce prediction. The basic model and its practical application are based on a model proposed in [12], developed and used in a major study of seven hundred newly married couples (see [20], [21] and for a survey [46]). The prediction of the future of marital stability proved surprisingly accurate, with an accuracy of 94 percent. Its use in marital therapy is proving highly successful.

Any mathematical or theoretical biological research must have genuine interdisciplinary content. There is no way mathematical modeling can solve major biological problems on its own. On the other hand, it is highly unlikely that even a reasonably complete understanding could come solely from experiment.

\section{References}

[1] P. Alberch, Developmental constraints in evolutionary processes, in J. T. Bonner, editor, Evolution and Development, Dahlem Conference Report, volume 20, Springer-Verlag, Berlin-Heidelberg-New York, 1982, 313-332.

[2] constraint in development and evolution, Geo. Bios., Mémoire Spéciale 12 (1988), 21-57.

[3] P. Alberch and E. Gale, Size dependency during the development of the amphibian foot, Colchicine induced digital loss and reduction, J. Embryol. Exp. Morphol. 76 (1983), 177-197.

[4] William L. Allen, Innes C. Cuthill, Nicholas E. SCOTT-SAMUEL, and ROLAND BADDELEY, Why the leopard got its spots: Relating pattern development to ecology in felids, Proc. R. Soc. B 278 (2011), 1373-1380.

[5] R. E. BAKER, S. SCHNELL, and P. K. MAINI, A clock and wavefront mechanism for somite formation, Dev. Biol. 293 (2006), 116-126.

[6] D. BERNOULLI, Essai d'une nouvelle analyse de la mortalité causée par la petite vérole, et des avantages de l'inoculation pour la prévenir, Histoire de l'Acad. Roy. Sci. (Paris) avec Mém. des Math. et Phys. and Mém. (1760), 1-45.

[7] S. BrenNer, J. D. MURRAY, and L. WOlPERT (editors), Theories of Biological Pattern Formation, Proceedings of the Royal Society meeting of that name at the Royal Society, London, 1981.

[8] J. Buhl, D. J. T. Sumpter, I. C. Couzin, J. Hale, E. DeSPLAND, E. MiLlER, and S. J. SIMPSON, From disorder to order in marching locusts, Science 213 (2006), 1402-1406.

[9] P. K. Burgess, P. M. Kulesa, J. D. Murray, and E. C. ALVORD JR., The interaction of growth rates and diffusion coefficients in a three-dimensional mathematical model of gliomas, J. Neuropathol Exp. Neurol 56 (1997), 704-713.

[10] S. CAmazine, J.-L. Deneubourg, J. Franks, J. SNeYd, GuY Theraulaz, and ERIC BonABEAU, Self-Organization in Biological Systems, Princeton University Press, 2001.

[11] M. A. J. Chaplain, G. D. Singh, and J. C. MaClaChLAN, On Growth and Form Spatio-temporal Pattern Formation in Biology, John Wiley and Son, Ltd., 1999.

[12] J. CoOK, R. TySON, K. A. J. White, R. Rushe, J. GotTman, and J. D. Murray, Mathematics of marital conflict: Qualitative dynamic mathematical modelling of marital interaction, J. Family Psychology 9 (1995), 110-130.

[13] G. C. Cruywagen, D. E. Woodward, P. Tracqui, G. T. BArtoo, J. D. Murray, and E. C. Alvord JR., 
The modeling of diffusive tumors, J. Biol. Systems 3 (1995), 937-945.

[14] P. D. DALE, P. K. MAINI, and J. A. SherratT, Mathematical modelling of corneal epithelial wound healing, Math. Biosci. 124 (1994), 127-147.

[15] R. A. FISHER, The Genetical Theory of Natural Selection, 1930 (reprint: Dover, New York, 1958).

[16] E. A. GaffneY, P. K. Maini, J. A. Sherratt, and S. TUFT, The mathematical modelling of cell kinetics in corneal epithelial wound healing, J. Theor. Biol. 197 (1999), 15-40

[17] R. A. Gatenby, K. Smallbone, P. K. Maini, F. Rose, J. AVERILL, R. B. NAGLE, L. WORRALL, and R. J. GILlIES, Cellular adaptations to hypoxia and acidosis during somatic evolution of breast cancer, Brit. J. Cancer 97 (2007), 646-653.

[18] B. T. Grenfell, O. G. Pybus, J. R. GoG, J. L. N. Wood, J. M. DAly, J. A. Mumford, and E. C. Holmes, Unifying the epidemiological and evolutionary dynamics of pathogens, Science 303 (2004), 327-332.

[20] J. M. Gottman, J. D. Murray, C. Swanson, R. Tyson, and K. R. SwANSON, The Mathematics of Marriage: Dynamic Nonlinear Models, MIT Press, Cambridge, MA, 2002.

[21] J. M. GotTman, C. Swanson, and J. D. Murray, The mathematics of marital conflict: Dynamic mathematical nonlinear modelling of newlywed marital interaction, J. Family Psychol. 13 (1999), 1-17.

[22] A. L. HodgKIN and A. F. HuXLeY, A quantitative description of membrane current and its application to conduction and excitation in nerve, J. Physiol. (Lond.) 117 (1952), 500-544.

[23] W. JÄGER and J. D. MurRAY (editors), Modeling of Patterns in Space and Time, Proceedings of a workshop of that name in Heidelberg, 1983, Springer-Verlag, Heidelberg, 1994.

[24] M. D. Johnston, C. M. Edwards, W. F. BODMER, P. K. MAINI, and S. J. CHAPMAN, Mathematical modeling of cell population dynamics in the colonic crypt and in colorectal cancer, PNAS 104 (2007), 4008-4013.

[25] J. KeEner and J. Sneyd, Mathematical Physiology, Springer-Verlag, 1998. (Second Edition in 2 volumes, Springer-Verlag, 2008.)

[26] W. O. Kermack and A. G. MCKENDRICK, Contributions to the mathematical theory of epidemics, Proc. R. Soc. Lond. A 138 (1932), 55-83.

[27] A. KolmogorofF, I. Petrovsky, and N. PiscounofF, Étude de l'équation de la diffusion avec croissance de la quantité de matière et son application à un problème biologique, Moscow University Math. Bull. 1 (1937), 1-25.

[28] S. Kondo, M. IWASHITA, and M. YAMAGUCHI, How animals get their skin patterns: Fish pigment pattern as a live Turing wave, Inst. J. Dev. Biol. 53 (2009), 851-856.

[29] Simon A. LeVIN (editor), Frontiers in Mathematical Biology, Springer-Verlag, Berlin, 1992.

[30] _ The problem of pattern and scale in ecology Ecology 73 (6) (1992), 1943-1967.

[31] M. A. Lewis, M. A. J. Chaplain, J. P. KeEneR, and P. K. MAINI (editors), Mathematical Biology, IAS/Park City Mathematics Series 14, Amer. Math. Soc., 2009.

[32] A. J. LOTKA, Elements of Physical Biology, Williams and Wilkins, Baltimore, 1925.

[33] Philip K. MAINI and HANS G. OTHMER (editors), Mathematical Models for Biological Pattern Forma- tion, Mathematics and its Applications, IMA Vol. 121, Springer, New York, 2000.

[34] Philip K. MAINI, How the mouse got its stripes, Proc. Nat. Acad. Sci. 100 (2003), 9656-9657.

[35] _ Using mathematical models to help understand biological pattern formation, C. R. Biologies 327 (2004), 225-234.

[36] H. MeInHARdT, Models of Biological Pattern Formation, Academic Press, London, 1982.

[37] J. D. Murray, Nonlinear Differential Equation Models in Biology, Clarendon Press, Oxford, 1977.

[38] _ A pre-pattern formation mechanism for animal coat markings, J. Theor. Biol. 88 (1981), 161-199.

[39] _ , On pattern formation mechanisms for lepidopteran wing patterns and mammalian coat markings, Phil. Trans. Roy. Soc. (Lond.) B 295 (1981), 473-496.

[40] J. D. Murray, G. F. Oster, and A. K. Harris, A mechanical model for mesenchymal morphogenesis. J. Math. Biol. 17 (1983), 125-129.

[41] J. D. MURRAY and G. F. OSTER, Generation of biological pattern and form, IMA J. Maths. Appl. in Medic. \& Biol. 1 (1984), 51-75.

[42] J. D. Murray and P. K. MAINI, A new approach to the generation of pattern and form in embryology, Science Progress 70 (1986), 539-553.

[43] J. D. MurraY, Mammalian coat patterns: How the leopard gets its spots, Scientific American 256 (1988), 80-87.

[44] _ Mathematical Biology, Springer-Verlag, Heidelberg, 1989.

[45] _ , Turing's theory of morphogenesis-its influence on modeling biological pattern and form, Bull. Math. Biol. 52 (1990), 119-152.

[46] _ Mathematical Biology. I: An Introduction, Springer, New York, 2002; Mathematical Biology. II: Spatial Models and Biomedical Applications (3rd edition in 2 volumes), Springer, New York, 2003.

[47] _ On the Growth of Brain Tumours: Enhancing Imaging Techniques, Highlighting Limitations of Current Imaging, Quantifying Therapy Efficacy and Estimating Patient Life Expectancy, in: Advances in Artificial Life, ECAL 2011: Proceedings of the Eleventh European Conference on the Synthesis and Simulation of Living Systems (eds. Tom Lenaerts, Mario Giacobini, Hugues Bersini, Paul Bourgigne, Marca Dorigo, René Doursat), MIT Press, 2011, pp. 23-26. (MIT Press online open-access proceedings volume: http://mitpress.mit.edu/catalog/item/ defau7t.asp?ttype $=2 \&$ tid $=12760$ )

[48] B. NABET, N. LeONARD, I. D. COUZIN, and S. A. LEVIN, Dynamics of decision making in animal group motion, Journal of Nonlinear Science 19 (4) (2009), 399-345.

[49] N. Frederick NiJhout, Philip K. Maini, ANotida MADZVAMUSE, ANDREW J. WATHEN, and TOSHIO SEKIMURA, Pigmentation pattern formation in butterflies: Experiments and models, C. R. Biologies 326 (2003), 717-727.

[50] G. F. Oster, J. D. Murray, and A. K. Harris, Mechanical aspects of mesenchymal morphogenesis, J. Embryol. Exp. Morph. 78 (1983), 83-125.

[51] G. F. OSTER, N. SHubin, J. D. MurRAY, and P. AlberCH, Evolution and morphogenetic rules: The shape of 
the vertebrate limb in ontogeney and phylogeney, Evolution 42 (1988), 862-884.

[52] Q. OUYANG and H. L. SWINNEY, Transition from a uniform state to hexagonal and striped Turing patterns, Nature 352 (1991), 610-612.

[53] K. J. PAinter, P. K. MAini, and H. G. OthmeR, Stripe formation in juvenile Pomacanthus explained by a generalized Turing mechanism with chemotaxis, Proc. Nat. Acad. Sci. 96 (1999), 5549-5554.

[54] D. I. RUbENSTEIN, Ecology, social behavior, and conservation in zebras, in: Advances in the Study Behavior: Behavioral Ecology of Tropical Animals (Macedo, R. ed.), Vol. 42, Elsevier Press, Oxford, UK, 2010, pp. 231-258.

[55] Geoffroy SAINT-Hilaire, Traité de Tératologie, Vols. 1-3, Baillière, Paris, 1836.

[56] J. A. SHERRATT, Mathematical Models of Wound Healing, D. Phil. Thesis, University of Oxford, 1991.

[57] J. A. SHERrATt and J. D. Murray, Epidermal wound healing: The clinical implications of a simple mathematical model, Cell Transplantation 1 (1992), 365-371.

[58] N. SHUBin and P. AlBerCH, A morphogenetic approach to the origin and basic organization of the tetrapod limb, in M. Hecht, B. Wallace, and W. Steere, editors, Evolutionary Biology, Volume 20, 319-387. Plenum, New York, 1986.

[59] N. Suzuki, M. HirAta, and S. Kondo, Traveling stripes on the skin of a mutant mouse, Proc. Nat. Acad. Sci. USA 100 (2003), 9680-9685.

[60] K. R. Swanson, E. C. Alvord JR., and J. D. Murray, Virtual brain tumors (gliomas) enhance the reality of medical imaging and highlight inadequacies of current therapy, Br. J. Cancer 86 (2002a), 14-18.61.

[61] _ , Quantifying the efficacy of chemotherapy of brain tumors with homogeneous and heterogeneous drug delivery, Acta Biotheoretica 50 (2002b), 223-237.

[62] D. Thomas, Artificial enzyme membranes, transport, memory, and oscillatory phenomena, in: Analysis and Control of Immobilized Enzyme Systems, D. Thomas and J.-P. Kernevez (editors), Springer-Verlag, BerlinHeidelberg-New York, 1975, 115-150.

[63] D'ARCY W. ThOMPSON, On Growth and Form, Cambridge University Press, 1917. (2nd edition, 1942.)

[64] A. M. TuRING, The chemical basis of morphogenesis, Phil. Trans. Roy. Soc. B237 (1952), 37-72.

[65] V. Volterra, Variazionie fluttuazioni del numero d'individui in specie animali conviventi (Variations and fluctuations of a number of individuals in animal species living together, translation by R. N. Chapman. In: Animal Ecology, McGraw Hill, New York, 1931, pp. 409-448; Mem. Acad. Lincei. 2 (1926), 31-113.

[66] A. T. WinfreE, Spiral waves of chemical activity, Science 175 (1972), 634-36.

[67] L. WOLPERT, Positional information and the spatial pattern of cellular differentiation, J. Theor. Biol. 25 (1969), 1-47.

[68] _ Principles of Development, Oxford University Press, 2006.

[69] A. M. ZHAвотINSKY, Periodical oxidation of malonic acid in solution (a study of the Belousov reaction kinetics), Biofizika 9 (1964), 306-11.
American Mathematical Society

\section{MATHEMATICAL} MODELLING TITLES

from the Applied Mathematics collection

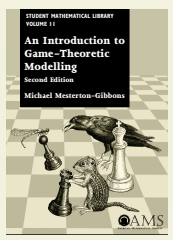

AN INTRODUCTION

TO GAME-THEORETIC MODELLING

\section{Second Edition}

Mike Mesterton-Gibbons, Florida State

University, Tallahassee, $F L$

An introduction to using game theory in mathematical modelling, covering the basic ideas and methods of game theory as well as the necessary ideas from the vast spectrum of scientific study where the methods are applied. The recurring theme is that game theory is fun to learn, doesn't require a large amount of mathematical rigor, and has great potential for application. This second edition contains a significant amount of updates and new material, including an important chapter on population games.

Student Mathematical Library,Volume II; 200I; 368 pages; Softcover; ISBN: 978-0-82 I8-1929-6; List US\$45;AMS members US\$36; Order code STML/II

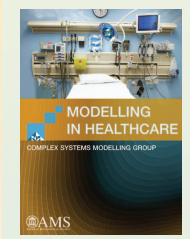

\section{MODELLING IN HEALTHCARE}

The Complex Systems Modelling Group (CSMG), The IRMACS Center, Simon Fraser University, Burnaby, BC, Canada

How many patients will require admission to my hospital in two days? How widespread will influenza be in my community in two weeks?

These and similar questions are the province of Modelling in Healthcare. This new volume ... uses plain language, sophisticated mathematics and vivid examples to guide and instruct ... the content and the logic are readily understandable by modelers, administrators and clinicians alike. This volume will surely serve as their common and thus preferred reference for modeling in healthcare for many years. - Timothy G. Buchman, Ph.D., M.D., FACS, FCCM

2010; 218 pages; Hardcover; ISBN: 978-0-82 I8-4969-9; List US\$69; AMS members US $\$ 55.20$; Order code MBK/74

\begin{tabular}{ll}
\hline courant 23 & MATHEMATICAL METHODS IN \\
\hline $\begin{array}{ll}\text { Mathematical } \\
\text { Methods in } \\
\text { Immunology }\end{array}$ & IMMUNOLOGY \\
& $\begin{array}{l}\text { Jerome K. Percus, Courant Institute } \\
\text { of Mathematics, New York, NY, and }\end{array}$ \\
& Department of Physics, New York University, \\
& NY
\end{tabular}

The complexity of the mammalian adaptive immune system calls for its encapsulation by mathematical models, and this book aims at the associated description and analysis. In the process, it introduces tools that should be in the armory of any current or aspiring applied mathematician, in the context of, arguably, the most effective system nature has devised to protect an organism from its manifold invisible enemies.

Titles in this series are co-published with the Courant Institute of Mathematical Sciences at New York University.

Courant Lecture Notes, Volume 23; 201 I; 1 II pages; Softcover; ISBN: 978-0-82 I 8-7556-8; List US\$32;AMS members US\$25.60; Order code $\mathrm{CLN} / 23$

\section{(i)AMS}

BOOKSTORE ams.org/bookstore

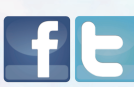

